\title{
Improving the attainment gap of Direct Entry Chinese students - lessons learnt and recommendations
}

\author{
Dawn Reilly, Wenxian Sun, Iwona Vellam, Liz Warren \\ University of Greenwich
}

\begin{abstract}
The expansion of Chinese articulations - which offer Chinese students the opportunity to study in the United Kingdom (UK) by permitting direct entrance into the final year of an undergraduate study programme - has provided a financially-attractive market. However, it has also raised concerns that these students do not have the same student experience as their peers and so may find it more difficult to attain a good degree classification. The culture shock - in terms of the impact of day-to-day life contrasts between the UK and China and the different pedagogical approaches of these two nations - creates additional barriers unique to these students. This case study therefore outlines experiences from two departments within a post-1992 Business School that has been recruiting students via articulations for thirteen years. The study highlights the journey towards adopting new approaches to teaching these students and thereby reducing the attainment gap. It thus offers an insight into which initiatives have proved successful and which ineffective and outlines plans to reduce the attainment gap further. The purpose of this study is to share our experience of creating conditions that support student success.
\end{abstract}

\section{Introduction}

Internationalisation of higher education $(\mathrm{HE})$ is a strategic initiative at many UK universities (Soliman et al., 2018). The Higher Education Statistics Agency (HESA) (2018) reports that more students from China than from any other overseas nationality study in the UK; nearly a third of all non-European Union (EU) students studying in the UK in 2016/17 $7^{1}$ were from China, with 102,772 Chinese students studying predominantly at Postgraduate (PG) level $\left(56 \%{ }^{2}\right)$, but with a significant proportion at undergraduate (UG) level $\left(38 \%{ }^{3}\right)$. The HESA (2018) report notes that the subject discipline which is most popular with the Chinese students is Business, recorded at $47 \%$. The three top sub-subject areas of the 18,185 Chinese students studying on UG business programmes are: Business Studies, with $29 \%$; Accounting, with $22 \%$, and Finance, also with $22 \%$.

This case study is set in the context of the Accounting and Finance (AF) and Systems Management and Strategy (SMS) departments within the Business School of a post-1992 university; however, we also outline some School-wide initiatives.

Both departments recruit some Chinese students to UG Years One and Two, but more of them to UG Year Three, under articulation agreements with Chinese institutions. In 2005, the Business School recruited from China eight Chinese Direct Entry students (CDEs), but, by

\footnotetext{
1 The 2018 HESA report provides data for the academic year 2016/17.

2 This includes taught and research.

3 The remaining $6 \%$ were reported as on non-degree programmes.
} 
2017, this number had increased to over 400. Here, we set out the extensive experience and insights we have, since 2005, gained from teaching CDEs and evaluate current practice regarding support for them.

\section{Literature review}

This case study highlights the attainment gap between Chinese students coming to the UK after studying in Chinese institutions and the student cohort in general. Studies on the attainment gap are not new; for example, McDuff et al. (2018) document the gap between the attainment of students from black and minority ethnic (BME) backgrounds and that of their white peers, according to an initiative carried out by Kingston University. Within the BME student population, McDuff et al. (op.cit.) note that Chinese students perform the best, albeit still less well than white students. However, they focused on UK-domiciled students, whose UK entry qualifications could be measured and for whom secondary education was comparable and language not an issue.

The expanding number of students from China studying in UK universities has attracted researchers to explore the unique characteristics of this body of students relative to the overall cohort. Unsurprisingly, Willis and Sedghi (2014) observe that Chinese students do not comprise a homogenous group. Nevertheless, they do face common challenges in the UK, such as the requirement to listen, read and write in English, their distance from family, culture shock, and 'learning culture shock'. Wilson (2009) emphasises how students are encouraged to embrace criticality and debate in the UK context, which differs dramatically form the rote-learning style to which they are accustomed. In a longitudinal study of Accounting and Finance UG students, Crawford and Wang (2015) observed that Chinese students outperformed UK students in Year One. However, this situation was reversed in Years Two and Three. Crawford and Wang (op.cit., p.917) explain that "... subjects in the second and final years demand deep and strategic learning approaches which Chinese students fail to develop". This finding presents a serious challenge to any HE institution recruiting Chinese students directly to Year Three of a UK degree programme.

Tran (2013) suggests that the Confucian tradition of passive learning, which prevails in China, can be overcome through exposure to a new and active approach, but this takes time and students require understanding and support during the transition. Recruiting students directly into Year Three means the opportunity to prepare them is very limited. Some examples of how we achieve this are set out in this paper. First, we explain how we have introduced measures to prepare CDEs for their study at a UK university before they enter the programme. Second, we set out our approach to teaching and assessment methods on Year Three direct entry programmes in an effort to reduce the Chinese attainment gap. Third, recognising the enriched learning environment which internationalisation affords to all students, we discuss how we have promoted positive and supportive interactions between Chinese students and their peers. Finally, we reflect on our current practice and set our plans for further improvement. 


\section{Background}

As previously mentioned, the Business School has been, since 2005, recruiting students through articulation ${ }^{4}$ agreements with China and, in 2017/18, recruited 428 new CDE students. The departments discussed in this case study face some common problems and some unique ones. The AF department has been offering articulations for longer, but takes on a smaller group of students than the SMS department. The accounting degree is highly technical and results in professional accreditation, so CDEs joining the AF department have to understand not only general business English but specific accounting language also. Meanwhile, the SMS department recruits the largest group of CDEs in the University (343 students in 2017/2018). The experiences of these two departments therefore provide a valuable knowledge resource concerning teaching strategies appropriate to the facilitation of student success. In addition, this study is highly relevant, as both departments teach the most popular subject areas selected by Chinese students: Business Studies, Accounting and Finance.

Within the two departments an attainment gap for CDEs remains, but it has been significantly reduced over time, to the benefit of both the CDEs and continuing students. In the AF department, where we have a specialised programme, the attainment gap in relation to good honours was, for $2017 / 18,16 \%{ }^{5}$, whereas six years ago it was $24 \%^{6}$. If we consider the more general business sister programme in the SMS department for those with an interest in accounting, there was an attainment gap of $1 \%$ in $2017 / 18$ compared to $18 \%$ three years $\mathrm{ago}^{7}$ when recruitment started. An attainment gap in the specialist programme therefore remains - if significantly reduced - and the attainment gap in the non-specialist programme has almost disappeared. However, we need, over the next few years, to monitor the attainment gaps to ensure continuation of the diminishing trend.

\section{Findings and discussion}

The case study reflects on six areas perceived as critical to reducing the attainment gap and improving the student experience for CDEs: activities before the students join the programme; the induction process; programme content; programme delivery; language used; and support given.

\section{1) Activities before joining the University}

In most cases, as part of Business School articulation agreements, we help to prepare CDEs by sending UK university lecturers to teach in China. This provides students with an opportunity to practise their language skills with UK teaching staff. Students also experience the pedagogical approach used in the UK, helping to move students' expectations away from passive learning (Tran, op.cit.). Although this has proved a useful approach, it does not address issues such as culture shock, the weather and food, all of which create anxiety upon

\footnotetext{
4 "An articulation agreement involves a formal link between the University of Greenwich and an external partner, providing a guarantee that one or more cohorts of students who achieve an agreed standard in a programme at the partner institution will be able to progress to a particular stage of an award-bearing programme at the University of Greenwich, provided there is a close curriculum 'fit' for articulation purposes. Students may gain an exit award from the partner at the point of transfer to the University, although this is not always the case." (http://www.gre.ac.uk/collaborations/prospective/partnership-models)

${ }^{5}$ It should be noted that the \% good honours classifications for the CDEs is still at an excellent level but is simply not as high as that for continuing students.

6 The earliest data set we could access.

7 Three years were used here as a comparison, because one of the programmes is only four years old.
} 
arrival in the UK. In 2017/18, therefore, as part of a School-wide initiative, we operationalised the so-called 'one term project', with the aim of allowing Chinese students to experience and reflect on the inter-cultural aspects of their learning alongside their subject-specific courses. For the project, we recruit students from China for a single semester, whilst they are still undertaking their studies in China, selecting those expected to return to the UK institution in the future to undertake a Year Three entry programme. They study English intensively for nine weeks and take Year One academic courses relevant to their Chinese major. In addition, they engage in weekly activities designed to introduce them to living in the UK. This initiative has proved popular, attracting 50 students in Term One and 130 in Term Two of the academic year 2017/18. We shall not know whether the initiative successfully alleviates the issues mentioned above until the students return for Year Three.

\section{2) Induction}

Both departments offer bespoke induction weeks to introduce CDE students to University systems, such as the Virtual Learning Environment (VLE) ${ }^{8}$ we provide, and to meet each other and their lecturers socially. We have also embedded some subject content into our induction period. This is supported by University Ambassadors, who were previously CDEs themselves. Induction weeks can be anxiety-provoking for CDEs, many of whom have never left China or their families before, and so ambassadors provide vital help with translation and offer a peer-to-peer support system.

In addition, with the assistance of ambassadors during induction, the AF module leaders introduce their final-year modules. This ensures that the CDEs understand the content and methods of assessment, allowing them to make an informed choice about which options suit their strengths and learning styles. For example, AF highlights that the Audit module requires a large amount of independent reading and that the Taxation module is heavily calculationbased. To enable students to perform well, the AF department also revises prior learning before the students begin their final-year modules. For example, revision of financial analysis is covered by an early Term One assessment and this presented problems for the CDE students. The induction team now, therefore, offers support in this area to enable CDEs to understand and participate in the module from the outset, improving assessment outcomes.

Provision of access to a supportive student community, which integrates CDEs into the existing cohort of students during induction, is vital. In the AF department, there is a teambased pedagogical approach used to connect the CDEs with the continuing students. However, in the SMS department, the CDEs receive support from the SMS Student Community, who work very closely with the Chinese students, offering mentoring services through various self-organised events (including 'Buddy talk' and Christmas and Chinese New Year celebrations).

\section{3) Programme content}

In both departments, programme content has been considered to ensure an inclusive approach. One example in the SMS department was the requirement for module leaders to update case studies used in their teaching to incorporate country-and culture-specific examples. For instance, when talking about online shopping and the related logistics and supply chain management issues, the case study of 'Black Friday' was changed to

\footnotetext{
${ }^{8}$ The dedicated training on VLE during induction is essential to closing the attainment gap, because it enables the CDE students to access information and material in the same way as continuing students.
} 
'Double 11 Day', the most popular online shopping day in China. The more familiar context meant CDEs understood the topic more readily.

In their original form, the AF specialised programme and the non-specialist SMS sister programme used an existing accounting module designed for professional body accreditation. The CDEs in the $\mathrm{SMS}^{9}$ department had experienced, prior to their studies, assessment and teaching styles that differed from those on the specialist AF programme. A new module was therefore designed to include the required level of content whilst also ensuring that diverse learning styles and previous experiences were accommodated.

\section{4) Delivery of the programme}

An active approach to teaching can be challenging for Chinese students, who might be described as "passive, shy and unwilling to ask questions or speak up in class" (Tran, op.cit., p.58). In the AF department, we have thus adapted how we teach CDEs, depending on the nature of the subject and the timing of the assessment. One example is the Strategic Financial Management module, which uses a team-based approach. Students work in groups for the entire year on a case study and are then expected to give presentations to other students. To improve the engagement and performance of the CDEs, the groups are constituted so that each comprises both Chinese and continuing students. Furthermore, the continuing students, following initial training, act as peer mentors to the Chinese students, helping them to communicate readily, for example via 'WhatsApp', and to understand expectations regarding the presentation. The continuing students also benefit, because many of the companies that we analyse have significant business interests in China and so they learn from the CDEs' knowledge of these companies.

By contrast, in some circumstances, the best strategy is to separate the CDEs and the continuing students. For example, for one technical module, which is examined by a threehour exam in January, the AF department realised the CDEs could not keep up sufficiently with the pace of teaching in the first term. In response to student feedback from both groups, therefore, the groups are now taught separately, in order not to disadvantage continuing students. The CDEs are taught by an experienced lecturer with qualifications in teaching international students in English and with the requisite professional qualifications to teach technical accounting. The style of delivery is therefore adapted, but the required content is still covered.

The use of the Mentimeter online questioning system has also proved useful in enhancing the CDEs' confidence. During Term One we realised that, in a lecture setting, only the continuing students asked questions. However, since our introduction of Mentimeter, the CDEs are now engaging. The use of an anonymous online questioning system removes the embarrassment of speaking out in front of other students. However, we find that, in Term Two, the students have more confidence and are happy to speak to the lecturer directly.

\section{5) Language}

Although staff try not to talk too fast and provide written materials and multi-media to facilitate communication, there are significant challenges to the teaching of CDEs in English, as they have spent a relatively short time in the UK. In the SMS department we therefore

\footnotetext{
${ }^{9}$ Note that the programme being discussed here only recruits CDEs.
} 
offer a language support group comprising students and Chinese-speaking staff members ${ }^{10}$. This group was formed to translate important announcements into Chinese and communicate with all CDEs. 'WeChat' is the number one Chinese multi-purpose messaging App (one billion monthly users) and is widely used among the students to access information promptly. The AF department also uses 'WeChat' within personal tutoring groups.

Plans to reduce the attainment gap further include use of Panopto ${ }^{11}$, which will allow students to replay lectures to enhance their language skills, thereby increasing their understanding of the content of the modules.

\section{6) Face-to-face support}

Both departments appoint CDE students as programme representatives across the different programmes, improving communication among students and between students and staff. However, the two departments employ different approaches to individual face-to-face support, owing to the difference in CDE numbers. In the AF department, we offer one-to-one appointments to all final-year students to discuss draft coursework in two of the core modules. Tutors encourage students to bring a friend if they do not feel comfortable attending alone and allow them to record meetings so they can replay them to manage language concerns. This has helped us address expectations about what is required from assessment in the final year. Initially, the meetings were voluntary, but they were more effective for Chinese students once they were made compulsory on the advice of colleagues who had more knowledge of the educational system in China. The department has since learnt to select its descriptions of activities carefully.

The AF department model, however, is not scalable for the SMS department because of the large number of CDEs within it. The model of support adopted instead is very effective and it involves having a reception desk managed by a Chinese-speaking staff member. Whenever the CDEs have problems or questions, they can access support and know what to expect. Student feedback shows the reception desk method has raised CDEs' satisfaction by boosting their confidence about learning in a different environment.

\section{Conclusion and limitations}

Awareness of the significant culture shock and different pedagogical approaches experienced by CDE students has informed how we move students from being passive learners to active learners (Tran, op.cit.). Tailoring teaching to account for the challenges this group faces has minimised the attainment gap and improved the student experience. However, although this case study has shared many approaches that could help to develop other programmes welcoming CDEs, it is important to note that neither the CDEs themselves, nor the programmes on which they study, are homogenous (Willis and Sedghi, op.cit.). Both the AF and SMS departments have had to adjust their approaches according to subject content and the number of students they recruit. In order to meet the needs of such particular sets of students as CDEs, we understand the continuing requirement to review course content, delivery and the student experience in today's HE environment. By doing so,

\footnotetext{
10 It is important to note that we have lecturers from many nationalities who can speak Chinese.

${ }^{11}$ Panopto is a video management system that can be used to pre-record or live-capture teaching activities.
} 
we can ensure that they have a good experience and the opportunity to gain a good degree classification.

Although this case study has provided a useful insight into the initiatives undertaken to close the attainment gap in the study contexts, there are some limitations. First, there may be other factors not identified that have contributed to the improved performance of the CDEs: for example, better awareness among lecturers of the needs of these students could have led to a re-orientation of teaching and the materials posted on the VLE, both of which might have had a pronounced effect on the CDEs. Second, the study has not clarified whether the profile of the Chinese students has changed over the three-year period considered: for example, we should expect more highly-qualified students to adapt more readily. Finally, interventions by Chinese institutions may have been implemented before the CDEs come to the UK, as these institutions are becoming increasingly familiar with the expectations of the UK educational system.

By adopting new approaches, introducing new initiatives and enabling staff acquisition of a deeper understanding of students' needs, we have improved the experience for both CDEs and continuing students. Although the Business School still has an attainment gap in some programmes with articulations, the gaps are closing. However, we also acknowledge that, for specialist programmes, it is unlikely that the gap can be eliminated completely, owing to the significant leap these students must take to adjust both academically and culturally to the UK. However, we shall continue to adapt our delivery to improve the student experience for all students, including CDEs, so that our students can achieve the best possible outcomes.

\section{Reference list}

Crawford, I. and Wang, Z. (2015) 'The impact of individual factors on the academic attainment of Chinese and UK students in higher education.' Studies in Higher Education, 40(5), 902-920. Available at: https://doi.org/10.1080/03075079.2013.851182 (Accessed: 2 February 2019 Month Year).

HESA (2018) Higher Education Student Statistics: UK, 2016/17 - Where students come from and go to study. Available at: https://www.hesa.ac.uk/news/11-01-2018/sfr247-highereducation-student-statistics/location (Accessed: 30 May 2018).

McDuff, N., Tatam, J., Beacock, O. and Ross, F. (2018) 'Closing the attainment gap for students from black and minority ethnic backgrounds through institutional change.' Widening Participation and Lifelong Learning, 20(1), 79-101. Available at: https://doi.org/10.5456/WPLL.20.1.79 (Accessed: 2 February 2019).

Soliman, S., Anchor, J. and Taylor, D. (2018) 'The international strategies of universities: deliberate or emergent?' Studies in Higher Education,. Available at: https://doi.org/10.1080/03075079.2018.1445985 (Accessed: 2 February 2019).

Tran, T.T. (2013) 'Is the learning approach of students from the Confucian heritage culture problematic?' Educational Research for Policy and Practice, 12, (1), 57-65. Available at: https://link.springer.com/article/10.1007/s10671-012-9131-3 (Accessed: 2 February 2019). 
Willis, I. and Sedghi, G. (2014) 'Perceptions and experiences of home students involved in welcoming and supporting direct entry $2^{\text {nd }}$ year international students.' Practice and Evidence of Scholarship of Teaching and Learning in Higher Education, 9(1), 2-17. Available at: http://community.dur.ac.uk/pestlhe.learning/ojs/index.php/pestlhe/article/view/96 (Accessed: 2nd February 2019).

Wilson, J.A. (2009) 'Getting the best out of your students through cultural appreciation multiculturalism in a British university business classroom setting.' Compass: Journal of Learning and Teaching, 1(1), 53-63. Available at: http://web-dev-

csc.gre.ac.uk/conference/conf64/doc... (Accessed: 2nd February 2019). 